\title{
Indeterminacy of intertemporal equilibria under imperfect competition ${ }^{\star}$
}

\author{
Leo Kaas \\ Department of Economics and Finance, Institute for Advanced Studies, Stumpergasse 56, \\ 1060 Vienna, AUSTRIA (e-mail: kaas@ihs.ac.at)
}

\begin{abstract}
Summary. It is known that overlapping generations models with imperfectly competitive firm may exhibit a continuum of stationary equilibria. The reason of this indeterminacy is that different price expectation functions of consumers lead to different objective demand functions against which firm maximize. All these expectation functions fulfil perfect foresight in the equilibrium, but they can be arbitrary off the equilibrium. In this paper it is shown that it is not this arbitrariness which is responsible for the indeterminacy, but that the continuum of stationary equilibria emerges even if expectation functions are rational.
\end{abstract}

Keywords and Phrases: Overlapping generations model, Imperfect competition, Indeterminacy, Expectations.

JEL Classificatio Numbers: D43, D84, D91.

\section{Introduction}

Macroeconomic models with imperfect competition have been investigated intensively over the past two decades. Most influentia in this literature are the articles of Hart (1982) and Blanchard and Kiyotaki (1987) who consider static models with three types of goods, labor, a consumption good, and a third commodity (money) which appears as an argument of the utility functions of consumers. These utility functions can be interpreted as indirect utility functions of a temporary equilibrium model in which consumers maximize their lifetime utility and hold fia money to save for future consumption. Consumers form expectations about future commodity prices, and these expectations enter the indirect utility function as well since they determine the real value of their money holdings

\footnotetext{
* I would like to thank Volker Böhm, Thorsten Hens and Paul Madden for helpful comments.
} 
(see e.g. Grandmont, 1983). In this temporary equilibrium interpretation, Hart's assumption that nominal money balances enter the utility function corresponds to the case of inelastic price expectations, while Blanchard and Kiyotaki's assumption that real money balances enter the utility function corresponds to unit elastic price expectations.

Rigorous intertemporal equilibrium versions of these models, mostly with an overlapping generation structure, have been studied by Benassy (1991), d'Aspremont, Dos Santos Ferreira and Gérard-Varet (1995), Chang (1991), Rivard (1994), and Schultz (1992). All these models restrict attention to rational expectations equilibria by assuming that expectations are correct, but also by assuming that they are perfectly inelastic, i.e. that they do not depend on the current price level. However, there is no reason why agents should use perfectly inelastic expectation functions. Rankin (1992) has shown that if agents use price expectations functions which depend on the current price level, a whole continuum of stationary equilibria emerges. The equilibrium in which agents use inelastic expectation functions is only one of them, but there are many other stationary equilibria which are characterized by different expectation elasticities and which are all ignored in the above literature. Kaas (1998) shows, for instance, that Schultz's result on the impossibility of involuntary unemployment is not robust to expectation functions which are not perfectly inelastic.

The reason of this indeterminacy ${ }^{1}$, is that in models with imperfect competition not only point expectations about future prices, but whole expectation functions are crucial since they determine the demand functions and thereby the payoff functions of imperfect competitors. In particular, since the elasticity of these demand functions determines the markup, the elasticity of price expectations turns out to be an important determinant of an intertemporal equilibrium. Multiple stationary equilibria emerge since agents may use expectation functions with different price elasticities, but which are all consistent with perfect foresight in the equilibrium. Expectations off the equilibrium path cannot be determined by a perfect foresight condition and are therefore arbitrary.

One might suspect that it is this arbitrariness of off-equilibrium expectations that is responsible for the indeterminacy, and that equilibria become determinate if off-equilibrium expectations are fixe rationally. Rankin (1997) suggests a definitio of rational expectations which he calls "fully rational expectations". He imposes the condition that agents "make correct forecasts with regard to all possible actions of imperfectly competitive firms", while "correct forecasts" mean that agents forecast equilibrium states for the future. Hence, in each possible state (whether a temporary equilibrium or not) agents forecast equilibrium states for the future. At hand of an example, Rankin shows that with this definitio of

\footnotetext{
${ }^{1}$ This usage of the term indeterminacy differs from the usual definitio in OLG models (cf. Chiappori and Guesnerie, 1991) stating that in each neighborhood of some (stationary) equilibrium there exists an infinit number of intertemporal equilibria. Here indeterminacy means moreover that in each neighborhood of a stationary equilibrium there exists an infinit number of other stationary equilibria.
} 
rationality, expectations elasticities are uniquely determined, and therefore also stationary equilibria are unique.

However, Rankin's example also uses the assumption that there are separated markets for young and for old consumers, which implies that the equilibrium price in the old consumers' market is independent of the behavior and thereby the expectations of young persons. It will be shown in this paper that it is this special assumption which is needed for Rankin's result, but that in general, i.e. if young and old consumers trade in one market, rational expectations equilibria are still indeterminate.

To show this, the next section introduces a general temporary equilibrium framework which extends the framework of Grandmont (1988) in order to include also overlapping generations models with imperfect competition. In this framework it will be distinguished between a perfect foresight equilibrium, in which agents forecast the future correctly along the equilibrium path, and a $\mathrm{ra}$ tional expectations equilibrium, in which agents also make correct forecasts in (hypothetical) situations off the equilibrium path. It will then be shown that even the stronger concept of rational expectations does generally not tie down unique rational expectations equilibria. These results will be derived from a simple characterization of rational expectations equilibria in the general framework in Section 3, and they will be applied to intertemporal Cournot-Walras equilibria in Section 4 and to a market game between consumers in a one-good pure exchange overlapping generations economy in Section 5.

\section{A general temporary equilibrium framework}

There is a state space $X$ whose elements contain all the relevant information about the allocation in a given period. There are agents (which shall be in all subsequent models overlapping generations of consumers living for two periods) who make intertemporal decisions and have to forecast the state in the next period. A forecast function $\psi: X \rightarrow X$ expresses how the expectation of an agent about the future state $x^{e}$ depends on the current state $x$, i.e. $x^{e}=\psi(x)$. A temporary equilibrium correspondence is a correspondence $\mathrm{T}: X^{X} \rightarrow X$, which expresses how the set of temporary equilibria $\mathrm{T}(\psi)$ depends on the forecast function $\psi$ of agents (which are all assumed to have the same forecast function). In general, such a temporary equilibrium correspondence may depend also on past states or other variables, but for the current purpose this formulation is sufficient

Assume that time starts at some initial date $t=0$ and that agents may use all past and current information when they form their forecasts. Hence, a full forecast function of the agents in period $t$ is a mapping $\Psi_{t}: X^{t+1} \rightarrow X$ with the meaning $x_{t+1}^{e}=\Psi_{t}\left(x_{t}, \ldots, x_{0}\right)$. The set of temporary equilibria in period $t$, given a full forecast function $\Psi_{t}$ and the past states $\left(x_{t-1}, \ldots, x_{0}\right)$, is then $\mathrm{T}\left(\Psi_{t}\left(., x_{t-1}, \ldots, x_{0}\right)\right)$. 
Definitio 1: $\left(x_{t}^{*}\right)_{t \geq 0}$ is a sequence of temporary equilibria generated by $\left(\Psi_{t}\right)_{t \geq 0}$ if for all $t \geq 0$

$$
x_{t}^{*} \in \mathrm{T}\left(\Psi_{t}\left(., x_{t-1}^{*}, \ldots, x_{0}^{*}\right)\right) \text {. }
$$

The next definitio imposes a perfect foresight condition on such sequences of temporary equilibria.

Definitio 2: $\left(x_{t}^{*}\right)_{t \geq 0}$ is a perfect foresight equilibrium (to be abbreviated PFE), if there is a sequence of forecast functions $\left(\Psi_{t}\right)_{t \geq 0}$ generating $\left(x_{t}^{*}\right)_{t \geq 0}$ as a sequence of temporary equilibria such that for all $t \geq 0$

$$
x_{t+1}^{*}=\Psi_{t}\left(x_{t}^{*}, \ldots, x_{0}^{*}\right)
$$

A PFE $\left(x_{t}^{*}\right)_{t \geq 0}$ is stationary if $x_{t}^{*}=x_{t+1}^{*}$ for all $t \geq 0$.

Clearly, $x_{t}^{*}=x^{*}, t \geq 0$, is a stationary PFE if and only if there exists $\psi: X \rightarrow X$ such that $\psi\left(x^{*}\right)=x^{*}$ and $x^{*} \in \mathrm{T}(\psi)$.

As explained in the introduction, expectations outside the equilibrium are an important determinant of a temporary equilibrium with imperfect competition. Definitio 2 imposes no restriction on these expectations, and one might suspect that this is a reason for indeterminacy of PFE. A possibility to defin "rationality" of forecast functions is to require that they forecast temporary equilibrium states whatever happened in the past. Formally:

Definitio 3: The sequence of forecast functions $\left(\Psi_{t}\right)_{t \geq 0}$, with $\Psi_{t}: X^{t+1} \rightarrow X$, $t \geq 0$, is called rational, if for all $\tau \geq 0$ and all $\left(x_{\tau}, \ldots, x_{0}\right) \in X^{\tau+1}$, the sequence of forecasts $\left(x_{t}\right)_{t>\tau}$ define recursively by

$$
x_{t}=\Psi_{t-1}\left(x_{t-1}, \ldots, x_{0}\right), t>\tau,
$$

describes temporary equilibria, i.e. it satisfie $x_{t} \in \mathrm{T}\left(\Psi_{t}\left(., x_{t-1}, \ldots, x_{0}\right)\right)$ for all $t>\tau$. A PFE, which is generated by a rational sequence of forecast functions, is called rational expectations equilibrium, to be abbreviated REE.

As an illustration of these definitions the following two examples show that in some cases a distinction between PFE and REE is superfluous while in others REE are a true selection of the set of PFE.

Example 1: Some perfectly competitive models (for instance, the standard onegood pure exchange OLG model) are described by a mapping $Z: X \times X \rightarrow \mathbb{R}^{n}$, where $X \subset \mathbb{R}^{n}$ (e.g. Chiappori and Guesnerie, 1991). A temporary equilibrium $x$ in a given period with expectations $x^{e}$ for the next period is define by the equation $Z\left(x, x^{e}\right)=0$. An intertemporal equilibrium (IE) in such a model is define as a sequence $\left(x_{t}^{*}\right)_{t \geq 0}$ fulfillin $Z\left(x_{t}^{*}, x_{t+1}^{*}\right)=0$ for all $t \geq 0$ (see e.g. Grandmont (1988)). Thus, an intertemporal equilibrium is define without using the concept of forecast functions. In the above framework, the temporary equilibrium correspondence is define by $\mathrm{T}(\psi)=\{x \in X \mid Z(x, \psi(x))=0\}$. It is clear that there are many forecast functions generating a given intertemporal 
equilibrium as a PFE in the sense of Definitio 2. Moreover, each intertemporal equilibrium is a REE in the sense of Definitio 3. Given an IE $\left(x_{t}^{*}\right)_{t \geq 0}$, the sequence of forecast functions $\Psi_{t}\left(x_{t}, \ldots, x_{0}\right):=x_{t+1}^{*}$ is rational and generates $\left(x_{t}^{*}\right)_{t \geq 0}$ as a REE. Thus, IE $\subset$ REE $\subset$ PFE. On the other hand, each PFE $\left(x_{t}^{*}\right)_{t \geq 0}$ clearly fulfill $Z\left(x_{t}^{*}, x_{t+1}^{*}\right)=0$ implying REE $\subset$ PFE $\subset$ IE. Therefore, the sets of PFE and of REE both coincide with the set of intertemporal equilibria, and off-equilibrium expectations do not matter.

Example 2: Consider a model with two-period living overlapping generations of consumers and two firms each producing at zero cost up to one unit of a single non-storable consumption good. Consumers consume in both periods of their life, have no endowment, receive all profi income when they are young, and save part of their income as fia money. Assume as Rankin (1997) that there are separated markets for young and for old consumers. Firm 1 is a monopolist on the market for young consumers, and fir 2 on the market for old consumers. Each old consumer holds an amount $M>0$ of fia money, and assume that fir 2 sets in each period the market clearing price $\bar{q}=M$ (actually fir 2 is indifferent between all prices $q \geq \bar{q}$ ). Assuming homothetic preferences, the young consumer's consumption demand can be written $c\left(\frac{q^{e}}{p}\right) \frac{I}{p}$ with a propensity to consume $0<c()<$.1 , where $p$ is the price of the consumption good, $I$ is his income, and $q^{e}$ is the expected price for the next period. Income consistency can be expressed $I=c\left(\frac{q^{e}}{p}\right) I+M$, implying that the income consistent demand of the young consumer is

$$
D\left(p, q^{e}\right):=\frac{M c\left(\frac{q^{e}}{p}\right)}{\left(1-c\left(\frac{q^{e}}{p}\right)\right) p}
$$

Assume that $c$ is strictly increasing, i.e. that consumption in both lifetime periods are gross substitutes. This implies that maximization of fir 1 's profi $D\left(p, q^{e}\right) p$ is equivalent to maximization of $q^{e} / p$. Let $\psi(p)=q^{e}$ be a price expectation function of the young consumer. Then fir 1 's decision problem is

$$
\max _{p>0} \frac{\psi(p)}{p} \quad \text { s.t. } \quad D(p, \psi(p)) \leq 1
$$

The state space $X$ is the set of prices $(p, q) \in \mathbb{R}_{++}^{2}$. With abuse of notation, identify $\psi$ with the second component of a full forecast function $\psi: X \rightarrow X$. Then the temporary equilibrium correspondence is

$$
\mathrm{T}(\psi):=\{(p, q) \mid p \text { solves (1) and } q=\bar{q}\} .
$$

Observe firs that PFE are completely indeterminate. For each $p_{t}$ satisfying $D\left(p_{t}, \bar{q}\right) \leq 1$, there exists a $\psi_{t}: \mathbb{R}_{++} \rightarrow \mathbb{R}_{++}$such that $\psi_{t}\left(p_{t}\right)=\bar{q}$ and $p_{t} \in \operatorname{argmax}_{p>0} \psi_{t}(p) / p$. Thus, $\left(p_{t}, \bar{q}\right) \in \mathrm{T}\left(\psi_{t}\right)$, and each sequence $\left(p_{t}, \bar{q}\right)_{t \geq 0}$ satisfying $D\left(p_{t}, \bar{q}\right) \leq 1$ for all $t$ is a PFE. 
In contrast, there exists only one REE. Since the only rational sequence of forecast functions $\left(\Psi_{t}\right)_{t>0}$ must forecast the price $\bar{q}$ irrespectively of the past ${ }^{2}$, the only solution of (1) with $\psi(.) \equiv \bar{q}$ is the unique $\bar{p}$ determined by $D(\bar{p}, \bar{q})=1$. Thus, the only REE is the constant sequence $(\bar{p}, \bar{q})_{t \geq 0}$.

\section{Characterization of rational expectations equilibria}

One might suspect that the above indeterminacy of PFE and stationary equilibria rests on the arbitrariness of forecast function away from the equilibrium states, and that, as in Rankin (1997) and the above Example 2, equilibria are determinate if forecast functions are rational. However, these models use the assumption of separated output markets for young and for old consumers, implying that the equilibrium price in the market for old persons is independent of the behavior and thereby the expectations of young persons.

By use of two examples, it will be shown now that the result of Rankin rests on this special assumption. If young and old persons trade in the same market, the equilibrium price in period $t+1$ depends on price expectations of young persons for period $t+2$. As a consequence, rationality of the forecast function in period $t$ depends on the forecast function in period $t+1$ whose rationality depends on the next forecast function, and so on. It turns out that these interrelations between forecast functions in different periods leads again to indeterminacy of REE.

To show indeterminacy of REE, a general result will be proven which characterizes REE by a simple geometric condition on sets of temporary equilibria. Formally, for $A \subset X$ let

$$
U(A):=\left\{\left(x, x_{+}\right) \in A \times A \mid \exists \psi: X \rightarrow A \text { with } x \in \mathrm{T}(\psi) \text { and } \psi(x)=x_{+}\right\} .
$$

Theorem 1. Let $A \subset X$ and let $g: A \rightarrow A$ fulfil $\operatorname{Graph}(g) \subset U(A)$. Then each trajectory of $g$, i.e. each sequence $\left(x_{t}^{*}\right)_{t \geq 0}$ with $x_{0}^{*} \in A$ and $x_{t+1}^{*}=g\left(x_{t}^{*}\right)$ for all $t \geq 0$, is a rational expectations equilibrium.

Proof. Let $x \in A$. Since $(x, g(x)) \in U(A)$, there exists $\psi(. ; x): X \rightarrow A$ such that $\psi(x ; x)=g(x)$ and $x \in \mathrm{T}(\psi(. ; x))$.

Let $\left(x_{t}^{*}\right)_{t \geq 0}$ be a trajectory of $g$. Defin $\left(\Psi_{t}\right)_{t \geq 0}$ recursively as follows:

$$
\Psi_{0}\left(x_{0}\right):=\psi\left(x_{0} ; x_{0}^{*}\right) \quad, x_{0} \in X,
$$

and for $t>0$

$$
\Psi_{t}\left(x_{t}, \ldots, x_{0}\right):=\psi\left(x_{t} ; \Psi_{t-1}\left(x_{t-1}, \ldots, x_{0}\right)\right) \quad,\left(x_{t}, \ldots, x_{0}\right) \in X^{t+1} .
$$

It has to be shown that $\left(\Psi_{t}\right)_{t \geq 0}$ is rational and that $\left(x_{t}^{*}\right)_{t \geq 0}$ is a PFE generated by $\left(\Psi_{t}\right)_{t \geq 0}$.

\footnotetext{
${ }^{2}$ Rankin (1997) considers a multi-sector model in which rational expectations can depend on the current price level. However, his model does not fi into the above framework, since the temporary equilibrium in period $t$ depends also on the distribution of money between sectors, and therefore also on the previous period's state.
} 
$\left(\Psi_{t}\right)_{t \geq 0}$ is rational: Let $\tau \geq 0$ and $\left(x_{\tau}, \ldots, x_{0}\right) \in X^{\tau+1}$. Defin $\left(x_{t}\right)_{t>\tau}$ recursively by $x_{t+1}=\Psi_{t}\left(x_{t}, \ldots, x_{0}\right)$ for all $t \geq \tau$. For $t>\tau$ it follows by construction

$$
\begin{aligned}
x_{t} & \in \mathrm{T}\left(\psi\left(. ; x_{t}\right)\right) \\
& =\mathrm{T}\left(\psi\left(. ; \Psi_{t-1}\left(x_{t-1}, \ldots, x_{0}\right)\right)\right) \\
& =\mathrm{T}\left(\Psi_{t}\left(., x_{t-1}, \ldots, x_{0}\right)\right)
\end{aligned}
$$

$\left(x_{t}^{*}\right)_{t \geq 0}$ is a PFE, i.e. $x_{t+1}^{*}=\Psi_{t}\left(x_{t}^{*}, \ldots, x_{0}^{*}\right)$ and $x_{t}^{*} \in \mathrm{T}\left(\Psi_{t}\left(., x_{t-1}^{*}, \ldots, x_{0}^{*}\right)\right.$ for all $t \geq 0$ : For $t=0$ it is, by definitio of $\psi(. ;),. x_{1}^{*}=g\left(x_{0}^{*}\right)=\psi\left(x_{0}^{*} ; x_{0}^{*}\right)=\Psi_{0}\left(x_{0}^{*}\right)$ and $x_{0}^{*} \in \mathrm{T}\left(\psi\left(. ; x_{0}^{*}\right)\right)=\mathrm{T}\left(\Psi_{0}().\right)$. For $t>0$ it is

$$
\begin{aligned}
x_{t+1}^{*} & =g\left(x_{t}^{*}\right)=\psi\left(x_{t}^{*} ; x_{t}^{*}\right) \\
& =\psi\left(x_{t}^{*} ; \Psi_{t-1}\left(x_{t-1}^{*}, \ldots, x_{0}^{*}\right)\right) \\
& =\Psi_{t}\left(x_{t}^{*}, \ldots, x_{0}^{*}\right) .
\end{aligned}
$$

Rationality of $\left(\Psi_{t}\right)_{t \geq 0}$ finall yields $x_{t}^{*} \in \mathrm{T}\left(\Psi_{t}\left(., x_{t-1}^{*}, \ldots, x_{0}^{*}\right)\right)$ for all $t>0$.

This theorem will be applied in the next sections to show the indeterminacy of REE in two examples. The general method is to fin appropriate subsets $A \subset X$ such that $U(A)$ is a "thick" set containing the graphs of many functions $g: A \rightarrow A$. Indeterminacy of stationary REE follows if there is a (non-trivial) subset $A$ such that $\operatorname{dia}(A):=\{(a, a) \mid a \in A\} \subset U(A)$, since then, by the above theorem, each $a \in A$ is a stationary REE.

\section{Intertemporal Cournot-Walras equilibria}

Consider an overlapping generations model in which discrete time $t$ goes from 0 to infinity and in which there are three commodities in each period: labor, a produced non-storable consumption good and money. There are $n \geq 2$ profit maximizing firm producing the consumption good by means of labor input with an (identical) convex labor requirement function $l: \mathbb{R}_{+} \rightarrow \mathbb{R}_{+}$. Firms know the objective (inverse) output demand and labor supply curves and act as Cournot oligopolists on both their output and their labor market. This type of competition is known as the Cournot-Walras equilibrium concept, as introduced by Gabszewicz and Vial (1972) in a static general equilibrium model.

There are representative overlapping generations of households living for two periods. They work and receive labor and profi income when they are young and no income when they are old, and they wish to consume in both periods of their life. Preferences of a representative young household are represented by a utility function of the type

$$
u\left(c_{1}, c_{2}\right)-v(L)
$$

Assumption. $u: \mathbb{R}_{+}^{2} \rightarrow \mathbb{R}$ and $v: \mathbb{R}_{+} \rightarrow \mathbb{R}$ are $C^{1}$ and strictly monotonic. $u$ is strictly quasi-concave and linearly homogeneous, and $u_{i}^{\prime}\left(c_{1}, c_{2}\right) \rightarrow \infty$ if $c_{i} \rightarrow 0, c_{j}>0, j \neq i$, for $i=1,2 . v$ is convex. 
Such types of utility functions have been used, for instance, by Hart (1982), Section 5, and by Dixon and Rankin (1994). The additive separability combined with linear homogeneity rules out any wealth effect on labor supply.

Let $\pi \geq 0$ denote the profi income of the young household, $w$ the wage rate, $p$ the actual goods price and $p^{e}$ the expected price for the next period. The solution of the decision problem

$$
\begin{aligned}
\max (2) \quad \text { s.t. } & p c_{1}+m \leq w L+\pi \quad, 0 \leq L \quad \text { and } \quad c_{1}, m \geq 0, \\
& p^{e} c_{2} \leq m \quad, c_{2} \geq 0 .
\end{aligned}
$$

fulfill

$$
\begin{aligned}
c_{1} & =c\left(\frac{p^{e}}{p}\right) \frac{w L+\pi}{p}, \\
c_{2} & =\left(1-c\left(\frac{p^{e}}{p}\right)\right) \frac{w L+\pi}{p^{e}}, \\
v^{\prime}(L) & =\frac{w}{\mathbf{P}\left(p, p^{e}\right)} \quad \text { if } 0<L,
\end{aligned}
$$

where $0<c()<$.1 is the propensity to consume, and where

$$
\mathbf{P}\left(p, p^{e}\right):=u\left(\frac{c\left(p^{e} / p\right)}{p}, \frac{1-c\left(p^{e} / p\right)}{p^{e}}\right)^{-1}
$$

is the intertemporal price index. Denote by $\psi: \mathbb{R}_{+} \rightarrow \mathbb{R}_{+}$a price forecast function, i.e. $p^{e}=\psi(p)$. If $Y$ is aggregate output and $L$ aggregate employment, aggregate profi income is $\pi=p Y-w L$ and the aggregate total income of the young household is $p Y$. Thus, the consumption demand of the young household is $c\left(p^{e} / p\right) Y$. The old household in period 0 holds an amount $M>0$ of money which remains constant over time, and therefore consumption demand of the old household is $M / p$. The output market equilibrium identity $c\left(\frac{\psi(p)}{p}\right) Y+\frac{M}{p}=Y$ can be solved for

$$
Y=\frac{M}{p(1-c(\psi(p) / p))} .
$$

Assume that $c$ and $\psi$ are such that this identity can be inverted, and write $p=P_{\psi}(Y)$ for the inverse demand function.

For each $L \geq 0$ the labor market clearing wage is $w=v^{\prime}(L) \mathbf{P}\left(p, p^{e}\right)$, and write

$$
W_{\psi}(Y, L):=v^{\prime}(L) \mathbf{P}\left(P_{\psi}(Y), \psi\left(P_{\psi}(Y)\right)\right)
$$

for the inverse labor supply function. Defin the profi function of a single fir if all other firm produce $y$ by $\pi_{\psi}\left(y^{\prime}, y\right):=y^{\prime} P_{\psi}\left((n-1) y+y^{\prime}\right)-W_{\psi}\left((n-1) y+y^{\prime},(n-1) l(y)+l\left(y^{\prime}\right)\right) l\left(y^{\prime}\right)$

Definitio 4: Let $\psi \in C\left(\mathbb{R}_{++}\right)$. A temporary symmetric Cournot-Walras equilibrium relative to $\psi$ is a tuple $(y, p, w)$ such that 


$$
\begin{aligned}
\text { (i) } y & \in \underset{y^{\prime} \geq 0}{\operatorname{argmax}} \pi_{\psi}\left(y^{\prime}, y\right), \\
\text { (ii) } & p=P_{\psi}(n y), \\
\text { (iii) } & w=W_{\psi}(n y, n l(y)) .
\end{aligned}
$$

The set of output prices in temporary symmetric Cournot-Walras equilibria relative to $\psi$ is denoted $\mathrm{T}(\psi)^{3}$

Notice that the implicit exclusion of inefficien production plans, i.e. not to consider production plans $(y, \ell)$ with $\ell>l(y)$, is no restriction here, since a reduction of the labor input does not increase the wage rate (because of convexity of $v$ ) and lowers therefore costs without changing revenue.

A rational expectations Cournot-Walras equilibrium is define as a REE in Section 2 for the state space $\mathbb{R}_{++}$and the above temporary equilibrium map $\mathrm{T}(\psi)$, and a stationary Cournot-Walras equilibrium is a stationary REE.

Consider for the rest of this section the following specificatio of the economy: $u$ is Cobb-Douglas with discount factor $(1-\alpha) / \alpha$, i.e.

$$
u\left(c_{1}, c_{2}\right)=\left(\frac{c_{1}}{\alpha}\right)^{\alpha}\left(\frac{c_{2}}{1-\alpha}\right)^{1-\alpha}
$$

such that

$$
c(.) \equiv \alpha \quad \text { and } \quad \mathbf{P}\left(p, p^{e}\right)=p^{\alpha}\left(p^{e}\right)^{1-\alpha} .
$$

Furthermore, assume

$$
\begin{aligned}
& v(L)=\frac{a}{\nu+1} L^{\nu+1} \quad, \nu \geq 0, a>0 \\
& l(y)=k \cdot y \quad, k>0 .
\end{aligned}
$$

This implies that the inverse output demand and labor supply functions are

$$
P_{\psi}(Y)=P(Y):=\frac{M}{Y(1-\alpha)} \quad, W_{\psi}(Y, L)=a L^{\nu} P(Y)^{\alpha}\left(\psi(P(Y))^{1-\alpha} .\right.
$$

It is quite useful to consider firs perfectly competitive equilibria where firm take the price and wage as given. That is, a temporary perfectly competitive equilibrium $(y, p, w)$ is a solution of $p=k w=P(n y)$ and

$$
w=a(n k y)^{\nu} p^{\alpha} \psi(p)^{1-\alpha} .
$$

Given a future output price $p_{+}$, it can be easily shown that there exists a unique temporary perfectly competitive equilibrium price $p$ such that $\psi(p)=p_{+}$. This price is given by

$$
p=p^{c}\left(p_{+}\right):=\left(a k^{1+\nu} p_{+}^{1-\alpha}\left(\frac{M}{1-\alpha}\right)^{\nu}\right)^{1 /(1-\alpha+\nu)} .
$$

Therefore, the function $p^{c}$ describes the backward perfect foresight dynamics under perfect competition. It is easy to verify that it has a unique fixpoin $\bar{p}^{c}$.

\footnotetext{
${ }^{3}$ Notice that the output price $p$ contains all relevant information: Given $p$, there is at most one $(y, w)$ such that $(y, p, w)$ is a temporary symmetric Cournot-Walras equilibrium.
} 
In contrast to the perfectly competitive case, rational expectations CournotWalras equilibria and also stationary Cournot-Walras equilibria are highly indeterminate. This will follow from the following theorem which shows that the sets $U(A)$ are large, provided that $A$ is an unbounded interval:

Theorem 2. Let $p_{0}>0$ and let $A:=\left[p_{0}, \infty\right)$. Then

$$
\left\{\left(p, p_{+}\right) \in A^{2} \mid p \geq p^{c}\left(p_{+}\right)\right\} \subset U(A) .
$$

The proof of this theorem needs the following two lemmas.

Lemma 1. Let $\left(p, p_{+}\right) \in \mathbb{R}_{++}$such that $p \geq p^{c}\left(p_{+}\right)$. Then there exists a continuous $\phi: \mathbb{R}_{++} \rightarrow \mathbb{R}_{++}$such that $p \in \mathrm{T}(\phi)$ and $p_{+}=\phi(p)$.

Proof. Let $\phi(P):=\mu P^{\gamma}$ where $\mu:=p_{+} p^{-\gamma}$, which implies $p_{+}=\phi(p) . \gamma \in \mathbb{R}$ will be chosen in the following such that $p \in \mathrm{T}(\phi)$.

A straightforward computation yields

$$
\pi_{\phi}\left(y^{\prime}, y\right)=\frac{M}{1-\alpha}\left(\frac{y^{\prime}}{y^{\prime}+(n-1) y}-B \frac{y^{\prime}}{\left(y^{\prime}+(n-1) y\right)^{\lambda}}\right)
$$

with

$$
B:=a k^{\nu+1} \mu^{1-\alpha}\left(\frac{M}{1-\alpha}\right)^{\alpha+\gamma(1-\alpha)-1} \text { and } \lambda:=\alpha+\gamma(1-\alpha)-\nu .
$$

Clearly, $y$ is the output of a single fir in a temporary symmetric Cournot-Walras equilibrium if it is a solution of the fixpoin problem

$$
y \in \underset{y^{\prime} \geq 0}{\operatorname{argmax}} \pi_{\phi}\left(y^{\prime}, y\right) .
$$

This solution of this problem can be computed as

$$
y \begin{cases}=\frac{1}{n}\left(\frac{B(n-\lambda)}{n-1}\right)^{1 /(\lambda-1)} & \text { if } \quad \lambda<1, \\ \in[0, \infty) \text { arbitrary } & \text { if } \lambda=1, B=1 .\end{cases}
$$

The corresponding temporary equilibrium price is then $\frac{M}{n y(1-\alpha)}$.

Assume firs that $p>p^{c}\left(p_{+}\right)$, and choose $\gamma$ such that

$$
\lambda:=\alpha+\gamma(1-\alpha)-\nu \stackrel{!}{=} n-(n-1)\left(\frac{p}{p^{c}\left(p_{+}\right)}\right)^{1-\alpha+\nu} .
$$

Notice that $\lambda<1$ because of $p>p^{c}\left(p_{+}\right)$. Thus, from (5), the temporary equilibrium price is

$$
\frac{M}{n y(1-\alpha)}=\frac{M}{1-\alpha}\left(\frac{B(n-\lambda)}{n-1}\right)^{1 /(1-\lambda)},
$$

which equals $p$ because of (6) and the definition of $B$ and $p^{c}$. Hence, $p \in \mathrm{T}(\phi)$.

Consider next the case $p=p^{c}\left(p_{+}\right)$and choose $\gamma$ such that $\lambda=1$. But now $p=p^{c}\left(p_{+}\right)$implies $B=1$ and it follows from (5) that $y=\frac{M}{n p(1-\alpha)}$ solves (4). Thus, $p \in \mathrm{T}(\phi)$. 
Lemma 2. Let $\psi, \phi: \mathbb{R}_{++} \rightarrow \mathbb{R}_{++}$and let $p>0$ such that $p \in \mathrm{T}(\phi), \psi(p)=\phi(p)$ and $\psi \geq \phi$. Then, $p \in \mathrm{T}(\psi)$.

Proof. Observe that $p \in \mathrm{T}(\phi)$ if and only if $y:=\frac{M}{n p(1-\alpha)}$ solves (4).

From $\psi \geq \phi$, it follows $\pi_{\psi} \leq \pi_{\phi}$, and $\phi(p)=\psi(p)$ yields $\pi_{\phi}(y, y)=\pi_{\psi}(y, y)$. Therefore, $y$ also solves (4) with $\pi_{\phi}$ replaced by $\pi_{\psi}$. Hence, $p \in \mathrm{T}(\psi)$.

Proof of Theorem 2. Let $\left(p, p_{+}\right) \in A^{2}$ such that $p \geq p^{c}\left(p_{+}\right)$. It has to be shown that there exists a continuous $\psi: \mathbb{R}_{++} \rightarrow\left[p_{0}, \infty\right)$ such that $p \in \mathrm{T}(\psi)$ and $p_{+}=\psi(p)$. Take $\phi$ from Lemma 1 and defin $\psi(P):=\max \left(\phi(P), p_{+}\right)$. Thus, $\psi(p)=p_{+}$and $\psi \geq \phi$. Lemma 2 implies that $p \in \mathrm{T}(\psi)$.

Corollary 1. Let $g:\left[\bar{p}^{c}, \infty\right) \rightarrow\left[\bar{p}^{c}, \infty\right)$ fulfil $g(p) \leq\left(p^{c}\right)^{-1}(p)$ for all $p \geq \bar{p}^{c}$. Then all trajectories of $g$ are rational expectations Cournot-Walras equilibria. In particular, each $\bar{p} \geq \bar{p}^{c}$ is a stationary Cournot-Walras equilibrium.

Proof. From the assumption, it follows $p \geq p^{c}(g(p))$ for all $p \geq \bar{p}^{c}$. Theorem 2 implies $\operatorname{Graph}(g) \subset U\left(\left[\bar{p}^{c}, \infty\right)\right)$ and Theorem 1 yields the firs claim. Since $p^{c}(p) \leq p$ for all $p \geq \bar{p}^{c}$, it follows $\operatorname{dia}\left(\left[\bar{p}^{c}, \infty\right)\right) \subset U\left(\left[\bar{p}^{c}, \infty\right)\right)$. Therefore, each $\bar{p} \geq \bar{p}^{c}$ is a stationary Cournot-Walras equilibrium.

Figure 1 illustrates $p^{c}(),. \bar{p}^{c}$, and one arbitrary function $g$ satisfying the requirement of Corollary 1.

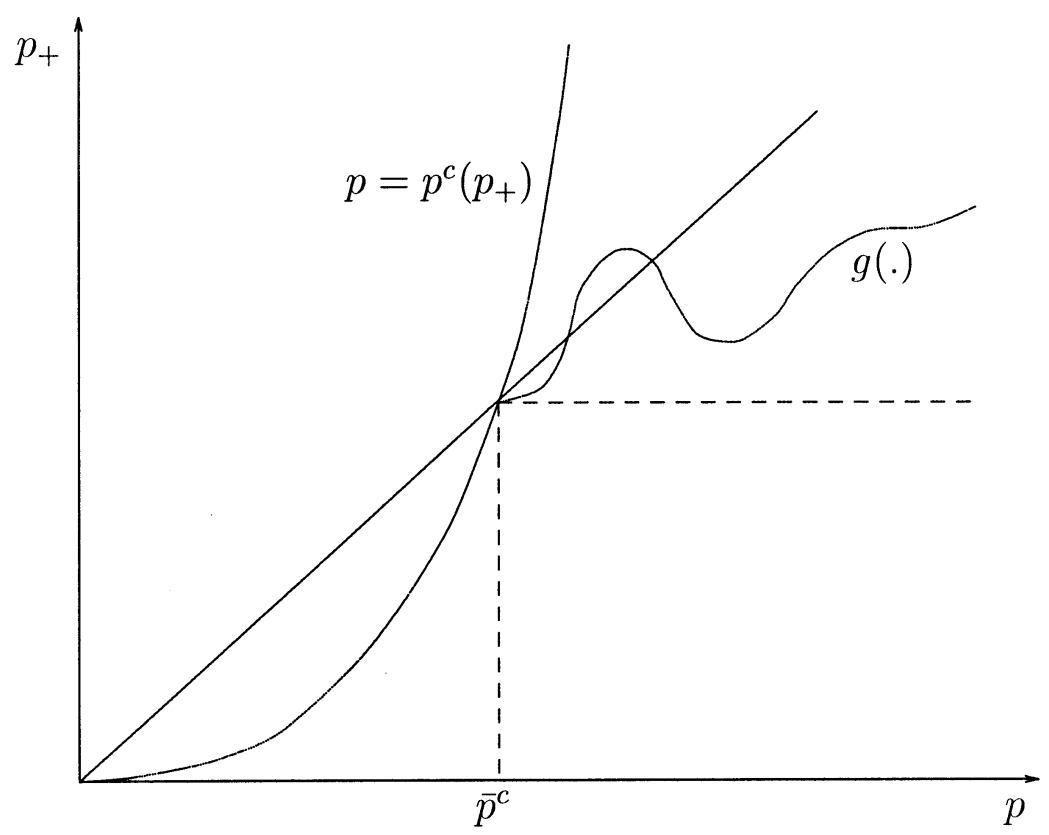

Figure 1. An arbitrary function $g$ generating rational expectations Cournot-Walras equilibria

A surprising implication of this result is that there is no convergence of rational expectations Cournot-Walras equilibria to perfectly competitive REE if 
the number of firm tends to infinity ${ }^{4}$ The set of REE obtained in Corollary 1 is independent of the number of firms Consider a REE $\left(p_{t}\right)_{t>0}$ for which $p_{t}>p^{c}\left(p_{t+1}\right)$ in one period. Then equation (6) in the proof of Lemma 2 implies that $\lambda$ and thereby the elasticity of the forecast function $\gamma$ tend to $-\infty$ as $n$ tends to infinity Therefore, steeper and steeper forecast functions are needed in order to support $\left(p_{t}\right)_{t \geq 0}$ as a REE. One would expect that convergence to perfectly competitive REE takes place if one imposes boundedness restrictions on the slopes of forecast functions.

One might suspect that the indeterminacy of REE is a consequence of the profi maximization hypothesis as it is the case for indeterminacies resulting from different choices of price normalization (see e.g. Grodal, 1996), and that this indeterminacy vanishes if shareholders decide on production plans and maximize their indirect utilities. However, this is not true. Assume that the single household is replaced by $n+1$ households, one worker and $n$ shareholders, each owning exactly one firm such that the worker has the same preferences as above over consumption and leisure, and such that the shareholders have the same preferences $u$ over consumption streams. It follows then from linear homogeneity of $u$ and separability of the worker's preferences that the inverse demand functions $P_{\psi}$ and $W_{\psi}$ are the same as before. Replace now the profi maximization hypothesis in (i) of Definitio 4 by the maximization of each shareholder's indirect utility which is

$$
U\left(y^{\prime}, y\right):=\frac{\pi_{\psi}\left(y^{\prime}, y\right)}{\mathbf{P}\left(P_{\psi}\left(y^{\prime}+(n-1) y\right), \psi\left(P_{\psi}\left(y^{\prime}+(n-1) y\right)\right)\right)}
$$

One can show easily with a modifie proof of Lemma 1 that Theorem 2 remains valid. The next section illustrates a similar indeterminacy result in a pure exchange economy.

\section{A market game in an OLG exchange economy}

Consider the following pure exchange overlapping generations model. There is a single non-storable consumption commodity and fia money. In each period $t=0,1, \ldots$ there are born $n$ two-period living consumers. Each consumer has an endowment $\omega>0$ of the consumption good when he is young, and zero endowment when he is old. Let $\Omega:=n \omega$ be the aggregate endowment in each period. Each of the $n$ old consumers in period 0 holds an amount $m>0$ of f at money. Let $M:=n m$ be the aggregate money endowment. Each consumer has the same utility function $u: \mathbb{R}_{+}^{2} \rightarrow \mathbb{R}$ representing preferences over consumption plans in their lifetime which is assumed to be strictly monotonic, strictly quasiconcave and continuous.

\footnotetext{
${ }^{4}$ In contrast, Kaas (1998) shows in an overlapping generations version of Hart's model convergence of all stationary PFE (and therefore also of all stationary REE) to the unique stationary perfectly competitive equilibrium.
} 
As proposed by Shapley and Shubik (1977), assume that in each period there is a trading post exchanging fia money for the consumption good. Assume that we are in a certain period and suppress for the moment the time index. Suppose that old consumer $i \in I:=\{1, \ldots, n\}$ in this period holds an amount $m_{i}$ of money.

Each young consumer $i \in I$ places at the trading post an offer $x_{i} \in[0, \omega]$, each old consumer places a bid $\mu_{i} \in\left[0, m_{i}\right]$ of money. All offers and bids are placed simultaneously. Given all offers and bids, there is a market clearing rule define in the following way. If $\mu:=\sum_{i \in I} \mu_{i}$ and $X:=\sum_{i \in I} x_{i}$ are both different from zero, there is trade at the market clearing price $p:=\mu / X$. If $X$ or $\mu$ is equal to zero, there is no trade. In this case, the young generation receives no money at all, and the economy becomes non-monetary and sticks to the no-trade equilibrium for all subsequent periods.

If $X>0$, it is a dominant strategy of each old consumer to bid all money. The old consumer $i$ maximizes consumption and solves

$$
\max _{0 \leq \mu_{i} \leq m_{i}} \frac{\mu_{i}}{\mu_{i}+\mu_{-i}} \cdot X
$$

which has the unique solution $\mu_{i}=m_{i}$ since $\mu_{-i}:=\sum_{j \neq i} \mu_{j}$ must be strictly positive, if other consumers maximize their consumption, too. Thus, each old consumer bids all money he owns, which implies that the aggregate money stock is equal to $M$ in all periods, as long as $X>0$ in all periods.

Each young consumer, knowing that he bids all money in the next period, forms expectations about the price in the next period. Since aggregate bids of old consumers are $M$, this is equivalent to forming expectations about the aggregate supply $X^{e}$ of young consumers in the next period. Assume that all young consumers expect that aggregate supply in the next period depends on aggregate supply $X$ in the current period according to a continuous forecast function $\psi:[0, \Omega] \rightarrow[0, \Omega]$, i.e. $X^{e}=\psi(X)$. The expected market clearing price is therefore $p^{e}=M / \psi(X)$.

If $\left(x_{i}\right)_{i \in I}$ is a list of offers, consumption of young consumer $j$ in the current period is $\omega-x_{j}$ and expected consumption in the next period is

$$
\frac{p x_{j}}{p^{e}}=\left\{\begin{array}{rll}
\frac{x_{j}}{\sum_{i \in I} x_{i}} \psi\left(\sum_{i \in I} x_{i}\right) & \text { if } & \sum_{i \in I} x_{i}>0 \\
0 & \text { if } & \sum_{i \in I} x_{i}=0
\end{array}\right.
$$

Definitio 5: Let $\psi \in C([0, \Omega]) . X \in[0, \Omega]$ is a temporary symmetric trading post equilibrium relative to $\psi$, either if

$$
0<\frac{X}{n} \in \underset{0 \leq \xi \leq \omega}{\operatorname{argmax}} u\left(\omega-\xi, \frac{\xi}{\xi+(n-1) \frac{X}{n}} \psi\left(\xi+(n-1) \frac{X}{n}\right)\right)
$$

or if

$$
0=X \in \underset{0 \leq \xi \leq \omega}{\operatorname{argmax}} u(\omega-\xi, \psi(\xi)) \quad .
$$

The set of temporary symmetric trading post equilibria relative to $\psi$ is $\mathrm{T}(\psi)$. 
This definitio cannot be immediately extended to a sequence of temporary equilibria as in Section 2. If $X=0$ is a temporary equilibria in one period, the economy becomes non-monetary and all temporary equilibria in subsequent periods must be no-trade equilibria. Thus, the above definitio can only describe sequences of temporary equilibria, as long as $X>0$ in all periods. However, under the following assumption on $u$ and $\psi$, a no-trade temporary equilibrium can be excluded.

Assumption. $u$ is continuously differentiable on $\mathbb{R}_{++}$and $\lim _{x \rightarrow 0} u_{2}^{\prime}(\omega, x)=\infty$, and $\psi$ is differentiable with $\psi^{\prime}(0)>0$.

Under this assumption, $0 \notin \mathrm{T}(\psi)$, since for small enough $\varepsilon>0$ it follows $u(\omega-\varepsilon, \psi(\varepsilon))>u(\omega, 0)$. A rational expectations trading post equilibrium is define as a REE in Section 2 for the state space $[0, \Omega]$ and the above temporary equilibrium map $\mathrm{T}(\psi)$, and a stationary trading post equilibrium is a stationary REE.

Theorem 3. Let $\Omega \geq C>0$ and $A=(0, C)$. Then

$$
\left\{\left(n x, n x_{+}\right) \in A^{2} \mid u\left(\omega-x, x_{+}\right)>u(\omega, 0)\right\} \subset U(A) .
$$

Proof. Let $0<x, x_{+}<c:=C / n$ and $u\left(\omega-x, x_{+}\right)>u(\omega, 0)$. One has to show that there exists $\psi:[0, \Omega] \rightarrow(0, C)$ such that $\psi(n x)=n x_{+}$and $n x \in \mathrm{T}(\psi)$.

Choose a continuous function $\Phi:[0, \omega] \rightarrow[0, c)$ such that

$$
\begin{gathered}
\Phi(x)=x_{+}, \\
u(\omega-\xi, \Phi(\xi))<u\left(\omega-x, x_{+}\right) \forall \xi \neq x \text { and } \Phi(\xi)<\xi \frac{c}{x} \forall \xi>0, \\
\Phi \text { is differentiable in } 0 \text { and } \frac{c}{x}>\Phi^{\prime}(0)>0 .
\end{gathered}
$$

Such a $\Phi$ exists as Figure 2 illustrates.

First, defin $\psi(X)$ for $(n-1) x<X \leq(n-1) x+\omega$ as follows:

$$
\psi(X):=\frac{\Phi(X-(n-1) x) X}{X-(n-1) x} .
$$

It follows $\psi(n x)=n x_{+}$and $\psi(X)>0$. If $X \leq n x, \Phi(\xi)<\frac{c}{X} \xi$ implies $\psi(X)<$ $\frac{c X}{x} \leq C$. If $X>n x, \Phi()<$.$c implies \psi(X)<\frac{C X}{n(X-(n-1) x)}<C$. By definitio of $\Phi, \psi$ can be continuously extended in $X=(n-1) x$ by $\psi((n-1) x)=$ $\Phi^{\prime}(0)(n-1) x \in(0, C)$. Therefore, $\psi(X) \in(0, C)$ for all $X \in[(n-1) x,(n-$ 1) $x+\omega]$.

Second, extend $\psi$ for $X \notin[(n-1) x,(n-1) x+\omega]$ arbitrary and continuous such that $0<\psi()<$.$C .$

Finally, if a consumer deviates to $\xi \in[0, \omega], x \neq \xi$, he gets worse off since

$$
u\left(\omega-\xi, \frac{\xi}{\xi+(n-1) x} \psi(\xi+(n-1) x)\right)=u(\omega-\xi, \Phi(\xi))<u\left(\omega-x, x_{+}\right) .
$$

Therefore, $n x \in \mathrm{T}(\psi)$. 


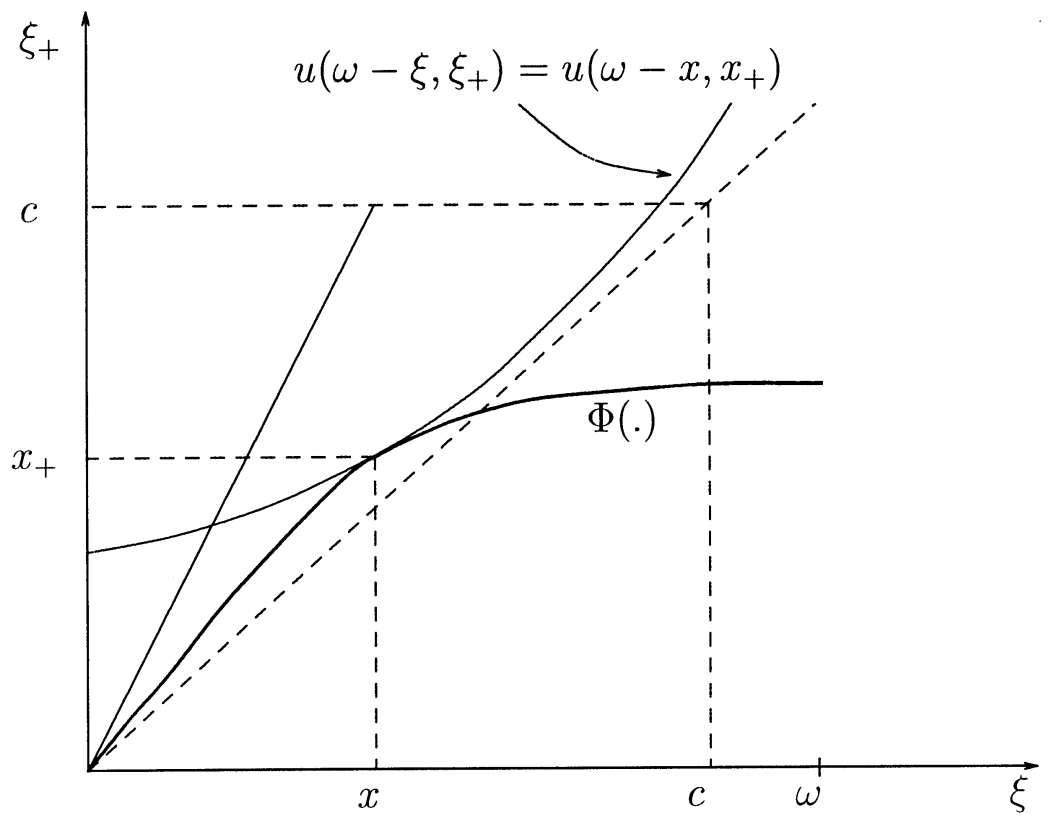

Figure 2. A function $\Phi$ fulfillin (7)

The following corollary shows that all individually rational trajectories are REE:

Corollary 2. Let $\bar{x}>0$ be chosen such that either $\bar{x}<\omega$ and $u(\omega-\bar{x}, \bar{x})=u(\omega, 0)$ or $\bar{x}=\omega$ and $u(0, \omega) \geq u(\omega, 0)$. Let $g:(0, \bar{x}) \rightarrow(0, \bar{x})$ fulfil $u(\omega-x, g(x))>$ $u(\omega, 0)$ for all $0<x<\bar{x}$. Then for each trajectory $\left(x_{t}^{*}\right)_{t \geq 0}$ of $g,\left(n x_{t}^{*}\right)_{t \geq 0}$ is a rational expectations trading post equilibrium. Furthermore, each $X \in(0, n \bar{x})$ is a stationary trading post equilibrium.

Proof. Defin $G(X):=n g(X / n)$. Theorem 3 implies $\operatorname{Graph}(G) \subset U((0, n \bar{x}))$. Theorem 1 proves the firs claim. Furthermore, $0<X<n \bar{x}$ implies $u(\omega-$ $\left.\frac{X}{n}, \frac{X}{n}\right)>u(\omega, 0)$ and thus $\operatorname{dia}(0, n \bar{x}) \subset U((0, n \bar{x}))$. This gives the second claim.

Figure 3 illustrates the set of individual rational allocations and one arbitrary function $g$ generating REE.

As in the previous section, there is no convergence of rational expectations trading post equilibria to perfectly competitive REE as $n$ tends to infinity since allocations of rational expectations equilibria are independent of $n$. Again, in the proof of Theorem 3, this may be attributed to the possibility of arbitrarily steep forecast functions. If the function $\Phi$ is differentiable in $x$, the forecast function is differentiable in $n x$ with slope

$$
\psi^{\prime}(n x)=\frac{x_{+}}{x}+n\left(\Phi^{\prime}(x)-\frac{x_{+}}{x}\right) .
$$

Observe that $\Phi^{\prime}(x)$ is equal to the marginal rate of substitution (see Figure 2): 


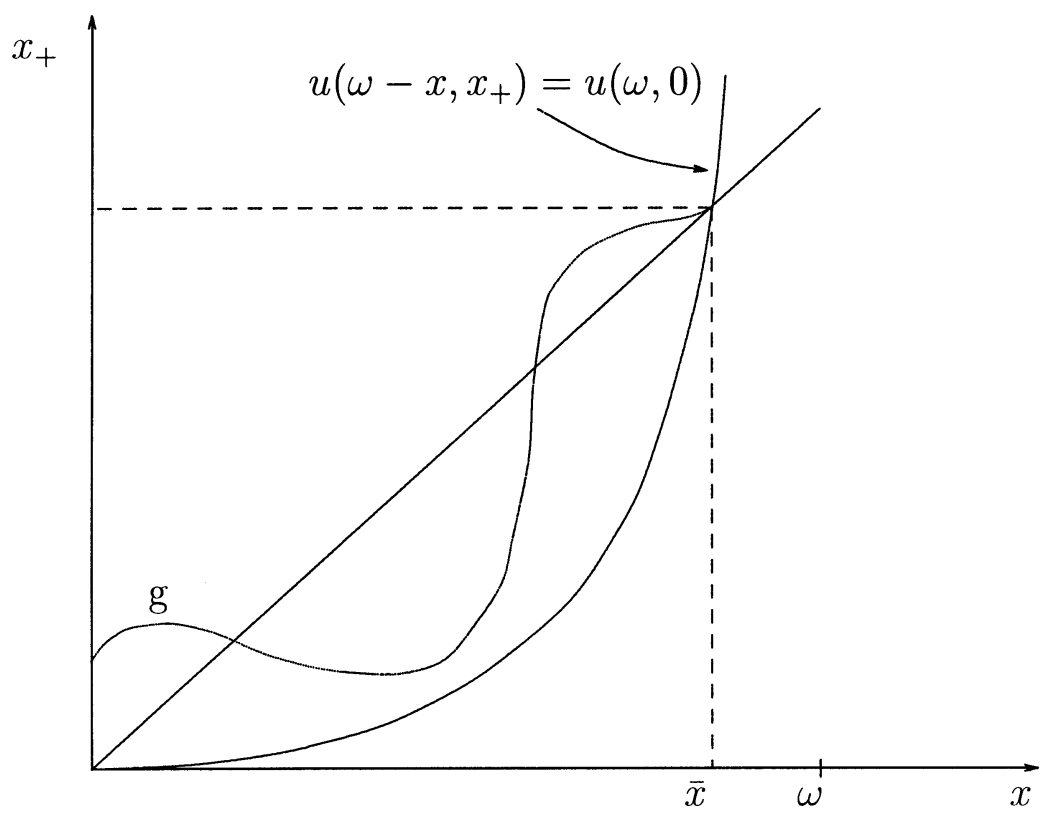

Figure 3. An arbitrary function $g$ generating rational expectations trading post equilibria

$$
\Phi^{\prime}(x)=\frac{u_{1}^{\prime}}{u_{2}^{\prime}}\left(\omega-x, x_{+}\right)
$$

Further, the offer curve, which describes price-taking behavior, is described by

$$
\frac{x_{+}}{x}=\frac{u_{1}^{\prime}}{u_{2}^{\prime}}\left(\omega-x, x_{+}\right)
$$

Therefore, if the pair $\left(x, x_{+}\right)$is not on the offer curve, the slope of the forecast functions supporting $x$ as a temporary equilibrium and forecasting $x_{+}$diverges if $n \rightarrow \infty$.

\section{Concluding remarks}

In this paper it has been shown that there is a serious indeterminacy problem in intertemporal models with imperfect competition. General equilibrium models with imperfect competition use the objective demand approach in order to avoid indeterminate equilibrium allocations due to subjective demand conjectures. Dynamic economic theory uses the rational expectations approach in order to avoid indeterminate solutions due to arbitrary expectation schemes. However, even though both the objective demand approach is used and all agents have rational expectations, the resulting intertemporal equilibria are highly indeterminate.

The explanation of this phenomenon is a kind of folk theorem argument. ${ }^{5}$ Different states in each period can be supported as a temporary equilibrium by

\footnotetext{
${ }^{5}$ There exist in fact folk theorems for repeated games with overlapping generations of players (Smith (1992) and Kandori (1992)), but these results cannot be applied here. Unlike folk theorems in
} 
some forecast function. But this forecast function is rational, if the states that are forecasted for the next period can itself be generated as temporary equilibria by the forecast function of the next period, and so on. Of course, if the economy ended in some finit period, the whole argument would break down.

\section{References}

Benassy, J.-P.: Optimal government policy in a macroeconomic model with imperfect competition and rational expectations. In: Barnett, W., Cornet, B., d'Aspremont, C., Gabszewicz, J., Mas-Colell, A. (eds.) Equilibrium theory and applications, pp. 339-352. New York Melbourne: Cambridge University Press 1991

Blanchard, O., Kiyotaki, N.: Monopolistic competition and the effects of aggregate demand. American Economic Review 77, 647-666 (1987)

Chang, R. (1991): Monopolistic competition, overlapping generations, and the role of monetary policy. Finnish Economic Papers 4, 24-32 (1991)

Chiappori, P., Guesnerie, R.: Sunspot equilibria in sequential markets models. In: Hildenbrand, W., Sonnenschein, H. (eds.) Handbook of Mathematical Economics, vol. IV, chap. 32. Amsterdam: North-Holland 1991

d'Aspremont, C., Dos Santos Ferreira, R., Gérard-Varet, L.: Imperfect competition in an overlapping generations model: a case for fisca policy. Annales d'Economie et de Statistique 37-38, 531-555 (1995)

Dixon, H., Rankin, N.: Imperfect competition and macroeconomics: a survey. Oxford Economic Papers 46, 171-199 (1994)

Gabszewicz, J. J., Vial, J. P.: Oligopoly 'à la Cournot' in general equilibrium analysis. Journal of Economic Theory 4, 381-400 (1972)

Grandmont, J.-M.: Money and value. Cambridge: Cambridge University Press 1983

Grandmont, J.-M.: Introduction. In: J.-M. Grandmont (ed.) Temporary equilibrium - selected readings. Boston: Academic Press 1988

Grodal, B.: Profi maximization and imperfect competition. In: Allen, B. (ed.) Economics in a changing world, vol. 2. London: MacMillan 1996

Hart, O. D.: A model of imperfect competition with Keynesian features. Quarterly Journal of Economics 97, 109-138 (1982)

Kaas, L.: Multiplicity of Cournot equilibria and involuntary unemployment. Journal of Economic Theory 80, 332-349 (1998)

Kandori, M.: Repeated games played by overlapping generations of players. Review of Economic Studies 59, 81-92 (1992)

Rankin, N.: Imperfect competition, expectations and the multiple effects of monetary growth. The Economic Journal 102, 743-753 (1992)

Rankin, N.: Imperfect competition and the modelling of expectations in macroeconomics. Working paper (1997), forthcoming in: Boitani, A., Salanti, A. (eds.) Information and coordination: a new Keynesian perspective. Dordrecht: Kluwer

Rivard, B.A.: Monopolistic competition, increasing returns, and self-fulfillin prophecies. Journal of Economic Theory 62, 346-362 (1994)

Schultz, C.: The impossibility of involuntary unemployment in an overlapping generations model with rational expectations. Journal of Economic Theory 58, 61-76 (1992)

Shapley, L., Shubik, M.: Trade using a commodity as a means of payment. Journal of Political Economy 85, 937-968 (1977)

Smith, L.: Folk theorems in overlapping generations games. Games and Economic Behavior 4, 426449 (1992)

game theory, the result here is based on variations of fundamentals of the economy, namely forecast functions. 